\title{
Substantiation of Electron-Seeking Catalysis of Vanadocene Dichloride Oxidative Breakdown in the Process of Vanadium Backwash from Heavy Karazhanbas Oilfield
}

\author{
RASHID I. BATKAYEV, ASIYA F. BATKAYEVA, \\ NIKOLAY N. ZOBNIN and ANASTASSIYA Y. KOVALEVA
}

M. Auezov South Kazakhstan State University, Kazakhstan, 160000, Shymkent, Tauke khan avenue, 5.

*Corresponding author E-mail: anastasiya2301@mail.ru

http://dx.doi.org/10.13005/ojc/320539

(Received: August 23, 2016; Accepted: October 15, 2016)

\begin{abstract}
In this article presents an extensive experimental results of the research of a vanadocene dichloride oxidative breakdown mechanism observable during the vanadium extraction from heavy oil. Particularly, an influence of sulfuric and nitric acid consumption on the process of demetallization of Karazhanbas oilfield heavy oil and kinetics of this reaction were considered. It was defined that the reaction will be carried out more effective in the case of substitution of sulfuric acid as an electrophilic catalyst for immobilized Lewis acids or mineral carrierimpregnated by hydrogen acids. Immobilized catalyst use allows to exclude involvement of nitric acid as an oxidizing agentin the process of vanadocene dichloride oxidative breakdown. Also the influence of ultrasonic influencepower during the oil emulsification and electroactivated aqueous solution to the kinetics demetallization were defined. New technological scheme were proposed, which provides "oil - electroactivated aqueous solution" emulsion preparation directly in oil-bearing strata. This engineering solution is able to reduce the number of process technological stages and to improve engineering-and-economical performance of demetallization.
\end{abstract}

Keywords: Vanadium, Electroactivated aqueous solution, Heavy oil, Oxidation-reduction potential, demetallization, productive solution.

\section{INTRODUCTION}

The synthesis of various organic compounds derivatives on the basis of vanadotcene is widely applied at creation of new catalytic systems for acceleration of homogeneous hydrogenation reactions of unsaturated compounds ${ }^{1}$. It would seem that the reverse reaction cannot lie in the sphere of researchers' interest. Attention to the reaction of oxidative decay vanadotsena with vanadium release in aqueous solution caused significant vanadium reserves of high-viscosity oil. The research proved, that crude oil contained about $10-12 \%$ of the vanadium in the aqueous component of the oil-in-water emulsion, i.e. in the form of soluble salts.Most of the vanadium $(90 \%)$ 
is concentrated in the organic component of the oilin-water emulsion. The form of vanadium in the oil particles, chemical reactions, likely in the system and mechanisms of their occurrence is very important. These parameters define the possibility and ways of vanadium extraction from crude oil, as well as an equipment used for this process and the main reactor in particular. There are many hypotheses concerning this matter. So according to the authors ${ }^{2}$ vanadium is in the elemental state or compounds in which vanadium forms a quasi-chemical bonds easy to break. According to these data leaching metals process from the crude oil on oilfields come to transfer of vanadium and nickel containing compounds from the oil emulsion to the salt solution at the expense of their interaction with a chemical reagent.

In the acid leaching of oxidized minerals vanadium transforms into solution in the form of vanadyl ion:

$3 \mathrm{~V}+6 \mathrm{HCl}+4 \mathrm{HNO}_{3} \rightarrow 3 \mathrm{VOCl}_{2}+4 \mathrm{NO} \uparrow+5 \mathrm{H}_{2} \mathrm{O}$

$3 \mathrm{~V}+3 \mathrm{H}_{2} \mathrm{SO}_{4}+4 \mathrm{HNO}_{3} \rightarrow 3 \mathrm{VOSO}_{4}+4 \mathrm{NO} \uparrow+5 \mathrm{H}_{2} \mathrm{O}$

On the one hand vanadyl sulfate is enough soluble in water and his formation could be explained the transfer of vanadium from the organic solution to aqueous solution. But on the other hand, according to the reference ${ }^{3}$ the reaction (2) is possible only with concentrated sulfuric acid at the temperature $90^{\circ} \tilde{\mathrm{N}}$ or by the reaction of ammonium metavanadate and ammonium phosphate in an acidic medium at the same temperature.Such conditions are not required in current methods of vanadium reextaction. Vanadium transfer to the aqueous solution carries outat the temperature $60-68^{\circ} \mathrm{C}^{4}$.

There is also a hypothesis that the vanadium is present in the oil as vanadyl porphyrin ${ }^{5}$. However, against this hypothesis is the fact that in the analysis of Karazhanbas and North Buzanchi oil samples by the method of $\mathrm{X}$-ray energy dispersive microanalysis revealed no adequate amounts of nitrogen. Taking into account small concentration of porphyrins, attempts were made to find the absorption spectrum in the between $1400-900 \mathrm{~cm}^{-1}$ corresponds to $\mathrm{C}-\mathrm{O}$, $\mathrm{C}-\mathrm{N}$ groups variations. However, such absorption bands were not found. But two absorption bands were found in the area of $1650-1600 \mathrm{~cm}^{-1}$, which indicates the presence of two conjugated $\mathrm{C}=\mathrm{C}$ bonds. According to the reference $e^{6-8}$ it could be an indication of vanadocene presents in the samples. Taking into account chloride background of aqueous solution, it must be assumed that the presence of the compound in the form of the dichloride.

There is also the data ${ }^{5}$ that the molecular weight of the oil entails increasing the share of nonporphyrin substances, and the share of porphyrin complexes decreases. Karazhanbas oil has a very high molecular weight, i.e. non-porphyrin substances should be considerable amount. So, in this oil vanadium should be in some non-porphyrin form.

\section{MATERIAL AND METHOD}

In order to research of the decay mechanism of the vanadocene dichloride process, the influence of proton acids (sulfuric and nitric acids) to the main indicators of demetallization was studied. To enhance the process effect, acids were dissolved in electrical activated water (subjected to electrolytic treatment). This water has a high positive redox potential up to +850-1200 mV. An additional oxidant contributes to the process of vanadocene dichloride oxidative decay. Oil was previously dehydrated in a centrifuge, weighed and calculated linkage fed into the reactor (5) for the demetallization (Figure 1).

The water, electrochemically activated in a reactor (1) was previously supplied to the reactor in the amount of $30 \mathrm{ml}$. Together with electrochemically activated water, $50 \%$ sulfuric acid, prepared in a reactor (2) and $50 \%$ nitric acid wee supplied to the main reactor. The reactor is a container, equipped by a heated jacket and mixer (6), driven by an electric motor (7), the rate was regulated by a laboratory autotransformer (8). The heating in the jacket was carried out by a hot water. The water was heated in a thermostat (9), then supplied by a pump to the reactor's jacket. The tests were carried out at temperatures of $70-80^{\circ} \mathrm{C}$. The temperature in the thermostat was regulated by a contact thermometer (15). After the decomposition, the pulp was supplied for a filtration. The filtration plant consisted of a Bunsen flask (11), filter funnel (10) and vacuum pump (12). The productive solution was delivered to the analysis, where it was analyzed for the presence of vanadium and nickel -containing products. A unit 
for determination of the oxidation-reduction potential consisted of $\mathrm{pH}$-meter (17) with calomel electrodes (15).

The solution after the filtration accumulated in a glass (16), where electrodes and a thermometer (18) were put in. For a uniform mixing the glass with the solution was installed on a magnetic stirrer (14). After the oxidation-reduction potential determination (17), the solution was supplied for the vanadium and nickel determination.

The investigation was carried out by a given below method. $100 \mathrm{~g}$ of the polymetallic raw material was placed into a reactor for the leaching, the preliminary electro-activated water in amount of $100-250 \mathrm{ml}$ was poured there. Then, the calculated amount of sulfuric acid and $\mathrm{NaCl}$ was added into the prepared solution.

The $\mathrm{pH}$ of the obtained productive solutions was 0.25 . Vanadium content in oil was determined according to State Standard 10398-76, and it amounted to $0.35 \%$. Waste gases were subjected to analysis to determine content of sulfur oxides, nitrogen and carbon by gas-analyzer «LIBET100 ».

Hydrodynamic mixing mode was maintained in all experiments at the upper boundary of turbulence with sub-critical speed. In the initial stages of research oil and electrical activated solutions mixing was performed using a laboratory shaker to obtain respective emulsions. At the final stage for the preparation of oil - activated water solution, bench-mountable ultrasonic bath «Y3K» line was used with volume $1.3 \mathrm{I}$. The bath has drowned piezoceramic transducers, operating capacity of steel, resistant to acids and alkalis, two-layer heatsaving cover, drain valve, a programmable timer, system of heating and maintaining the temperature, with remote generator. To obtain the lowest possible particle emulsion, ultrasound of ultra-high frequency was used - $5 \mathrm{MHz}$.

Research of the oil for the metal presence in its composition was carried out by atomic absorption spectrometry. The following devices, reagents and laboratory were used in research: ANALYST-800 atomic absorption spectrophotometer, completed with options for flame atomization of samples; standard solution of metals; TC-1 fuel; flask with $100 \mathrm{ml}$. Concentrated solution of the organic metal compounds was prepared (based on 100 ppm$^{-1}$ of each metal). Working standards were prepared immediately prior to analysis by serial dilution concentrate of fuel. Standard solutions were prepared with metal content: 0.02; 0.05; 0.10; 0.20; $0.50 ; 1.0 ; 2.0 ; 5.0$ and $10.0 \mathrm{ppm}^{-1}$. An average sample of oil (petroleum) was selected. In the $100 \mathrm{ml}$ flask the sample were taken diluted (in volume) in 5, 10 or 20 times by the fuel. It is necessary to take into account the density of the fuel. If the oil sample (or oil product sample) had a poor fluidity, it was heated.

Standard solutions and solutions were analyzed spectrophotometrically at the combustion in the air mixture in the flame-acetylene or nitrogen oxide (II) - acetylene. Analytical vanadium line installed at the exit slit of the spectrophotometer. The optimal parameters, which must be followed during the tests for vanadium and nickel determination in the oil composition are shown in Table 1.

Research of the emulsion microstructure in the oil-electroactivated solution system was carried out with using the following equipment: scanning electron microscope JSM 6490LV (JEOL, Japan) with the system of energy dispersive microanalysis INCA Enerjy-350 (OXFORD Instruments, UK) and the system of structural analysis of polycrystalline objects HKL Basis (OXFORD Instruments, UK ). The increase was carried out from 1000 to 10000 times. At the same time the content of chemical elements from boron to uranium was analyzed. Developed method of emulsion samples preparation saving the particle size of the aqueous and organic phases in a state of initial emulsification was applied. The methodology included the work in low vacuum mode, which gave additional opportunities for the most correct evaluation of emulsion particle size.

\section{RESULTS AND DISCUSSION}

In the result of research it was defined that vanadocene has a free electron or labile pairs in the form of unsaturated "carbon - carbon" bonds. In this respect, the double bond is the electron donor for the vanadium (which sends s-electrons to chlorine). Attack of protons proceeds on double 


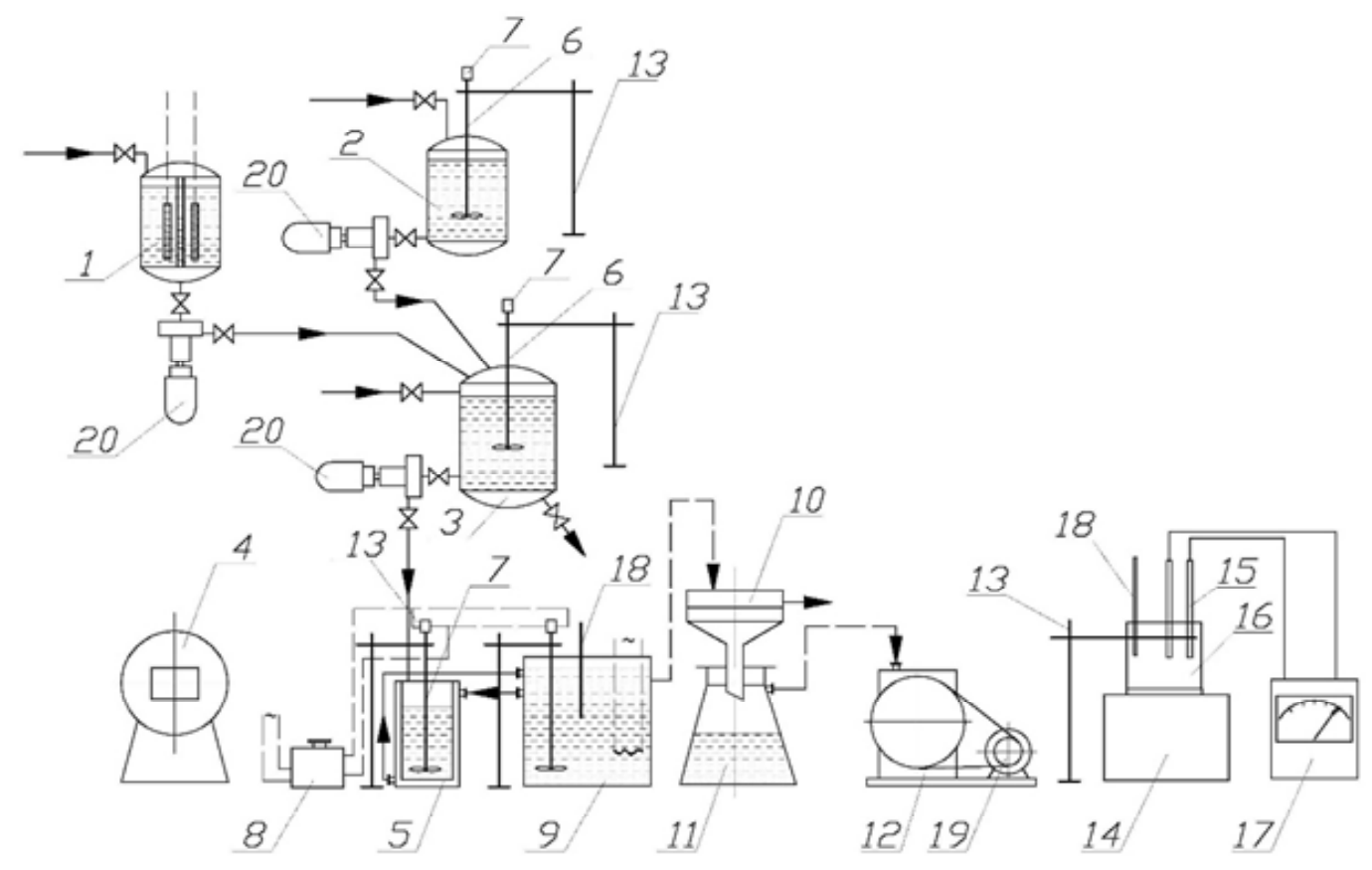

1 - activator; 2 - salt solution tank No. 1; 3 - salt solution tank No. 2; 4 - drying chamber; 5 - reactor; 6 - mixer; 7 - electromotor; 8 -laboratory autotransformer; 9 - thermostat; 10 - funnel; 11 - Bunsen flask; 12 - vacuum pump; 13 - holder; 14 - magnetic stirrer; 15 - electrodes; 16 - glass; 17 - pH-meter; 18 - control thermometer; 19 - electric motor; 20 - pump

Fig. 1: A scheme of the laboratory unit for the demetallization of oil stocks

Table 1: Optimal Parameters of The Absorption Measurement

\begin{tabular}{lcc}
\hline Indices & Vanadium & Nickel \\
\hline The wavelength of the analytical line, $\mathrm{nm}$ & 318,4 & 341,3 \\
Current lamp with a full cathode, $\mathrm{mA}$ & 11 & 8 \\
The slit width, $\mathrm{m}$ & 160 & 40 \\
The voltage on the PMT, W & 620 & 700 \\
Scale signal amplifier & 1 & 2,5 \\
Fuel & Acetylene & Acetylene \\
Oxidizer & Nitrogen oxide (II) & Air \\
The distance from the burner to the optical axis of the device, mm & 7 & 11 \\
Acetylene pressure, psig (0,007 MPa) & 6 & 5 \\
Oxidant pressure, psig & 0,5 & 6 \\
The sample flow rate, $\mathrm{ml} /$ min & 6 & 6 \\
Integration time, sec & 10 & 10 \\
\hline
\end{tabular}


$C=C$ bond. In this case, the electron density of the vanadium atom moves to the hydrogen proton. Bond of cyclopentadiene ring with vanadium destroyed. Simultaneously with the proton attack the double $\mathrm{C}=\mathrm{C}$ bond is attacked by $\mathrm{O}^{2-}$ ion. As a result, $\mathrm{C}=\mathrm{C}$ double bond breaks, stands $\mathrm{VCl}_{2}+$ ion and unprotonated molecule cyclopentenol are distinguished.
As the oxidizing agent in this reaction acts $\mathrm{O}^{2-}$ ion formed in the cathode space during the electrolysis (electroactivation) or ozone water formed in the anode compartment $\mathrm{NO}_{3}^{-}$or ions and/or $\mathrm{SO}_{4}{ }^{2-}$, formed by electrolytic dissociation. Vanadium included in $\mathrm{VCl}_{2}^{-}$ion in an oxidizing atmosphere is oxidized to the tetravalent state and appends hydroxyl ions from water. During this process vanadium dihydroxochloride is formed and

Table 2: The results of research of sulfuric and nitric acid consumption for the process of Karazhanbas oilfield demetallization process

\begin{tabular}{|c|c|c|c|c|}
\hline Indicators & $\underset{1}{\text { Sample }}$ & $\begin{array}{l}\text { Sample } \\
\quad 2\end{array}$ & $\begin{array}{c}\text { Sample } \\
3\end{array}$ & $\begin{array}{l}\text { Sample } \\
4\end{array}$ \\
\hline Oil weight, $g$ & 50,8 & 50,8 & 101 & 101 \\
\hline Activated water weight, $\mathrm{g}$ & 30 & 30 & 30 & 30 \\
\hline Volume of sulfuric acid $50 \%, \mathrm{ml}$ & 10 & 5 & 10 & 10 \\
\hline Volume of nitric acid $50 \%, \mathrm{ml}$ & 0 & 5 & 5 & 10 \\
\hline Concentration of sulfuric acid for total volume of emulsion, $\%$ & 7,36 & 3,97 & 4,81 & 4,81 \\
\hline $\begin{array}{l}\text { Concentration of nitric acid for total volume of emulsion, \% } \\
\text { Concentrations }\end{array}$ & 0 & 3,75 & 2,38 & 4,55 \\
\hline Vanadium in oil, \% & 0,35 & 0,35 & 0,35 & 0,35 \\
\hline Vanadium in productive solution, \% & 0,335 & 0,113 & 0,637 & 0,6 \\
\hline Nickel in productive solution, \% & 0,16 & 0,18 & 1,89 & 2,03 \\
\hline Sulfur in productive solution, $\%$ & 3,12 & 1,12 & 2,96 & 1 \\
\hline Sulfur in oil, \% & 1,9 & & & \\
\hline Terminal weight of productive solution, $\mathrm{g}$ & 43,95 & 43,52 & 50,50 & 57,05 \\
\hline Initial weight of the vanadium in oil, $\mathrm{g}$ & 0,17 & 0,17 & 0,35 & 0,35 \\
\hline Terminal weight of vanadium in productive solution, $\mathrm{g}$ & 0,14 & 0,05 & 0,32 & 0,34 \\
\hline Recovery degree of vanadium to the productive solution, \% & 83 & 28 & 91 & 97 \\
\hline Sulfur weight in oil initial, g & 0,96 & 0,96 & 1,91 & 1,9 \\
\hline Sulfur weight in productive solution terminal, $\mathrm{g}$ & 1,37 & 0,48 & 1,49 & 0,57 \\
\hline Sulfur weight in productive solution initial, g & 2,27 & 1,14 & 2,27 & 2,27 \\
\hline Sulfur losses from sulfuric acid with oil, g & 0,09 & 0,29 & 0,30 & 1,60 \\
\hline Sulfur losses from sulfuric acid with oil, \% & 3,80 & 25,58 & 13,30 & 70,12 \\
\hline Sulfur weight in oil with a glance of income, $\mathrm{g}$ & 1,87 & 1,61 & 2,70 & 3,62 \\
\hline Finite sulfur concentration in oil, \% & 3,68 & 3,18 & 2,67 & 3,59 \\
\hline $\begin{array}{l}\text { Sulfuric acid consumption to } 1 \mathrm{~kg} \text { of vanadium in } \\
\text { productive solution, } \mathrm{kg}\end{array}$ & 18,85 & 40,56 & 7,45 & 15,27 \\
\hline $\begin{array}{l}\text { Nitric acid consumption to } 1 \mathrm{~kg} \text { of vanadium in } \\
\text { productive solution, } \mathrm{kg}\end{array}$ & 0 & 66,6 & 10,2 & 19,1 \\
\hline Sulfur in gases, $\mathrm{g}$ & 0,82 & 0,36 & 0,48 & 0,11 \\
\hline Nitrogen in gases, $\mathrm{g}$ & 0,00 & 0,73 & 0,73 & 1,46 \\
\hline Gas volume separated in the process, I & 0,57 & 1,42 & 1,50 & 2,41 \\
\hline Finite oil weight theoretical, $g$ & 50,49 & 50,04 & 100,20 & 99,71 \\
\hline Oil losses in the process of demetallization (theoretical), \% & 0,61 & 1,49 & 0,80 & 1,28 \\
\hline
\end{tabular}


two hydrogen protons are regenerated, which enter the process again. This mechanism is confirmed by IR spectroscopy. In the spectrogram disappears one of the two absorption bands in the $1650-1600 \mathrm{~cm}^{-1}$, indicating that the decay of one of the conjugated $\mathrm{C}=\mathrm{C}$ bonds. At the same time there are characteristic absorption bands in the regions $3500-3600 \mathrm{~cm}^{-1}$ (stretching vibrations of $\mathrm{OH}$ - groups) and 1400-1000 $\mathrm{cm}^{-1}$ (fluctuations associated with the group $\mathrm{C}-\mathrm{O}-\mathrm{H}$ ). The stretching vibrations of $\mathrm{O}-\mathrm{H}$ are characteristic because they are attended by a light hydrogen atom. Since there are no conditions for the association of molecules, then the spectrogram recorded only a narrow absorption band at $3670-3580 \mathrm{~cm}^{-1}$ in cyclopentenol solution in oil (non-polar solvent).

In the absorption spectrum of the band also appeared in the area $950-1200 \mathrm{~cm}^{-1}$. From this it follows that a portion of sulfuric acid was consumed for adverse reactions of sulfation. So sulfuric as

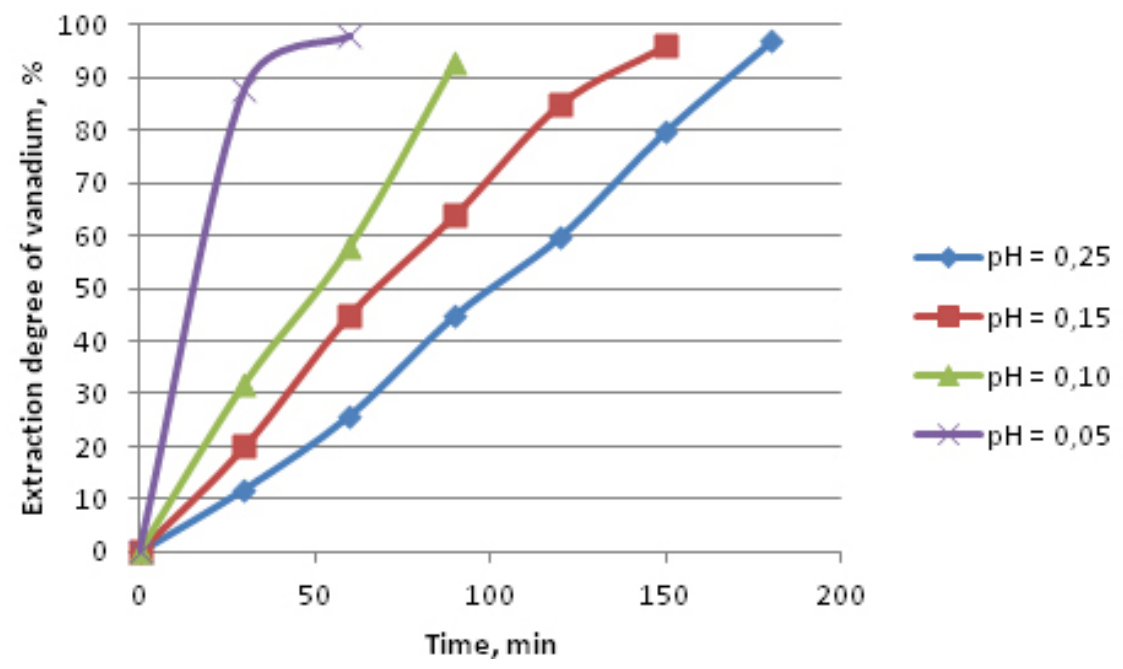

Fig. 2: The dependence of vanadium extraction degree from the thermostating time and the $\mathrm{pH}$ of the final productive solution

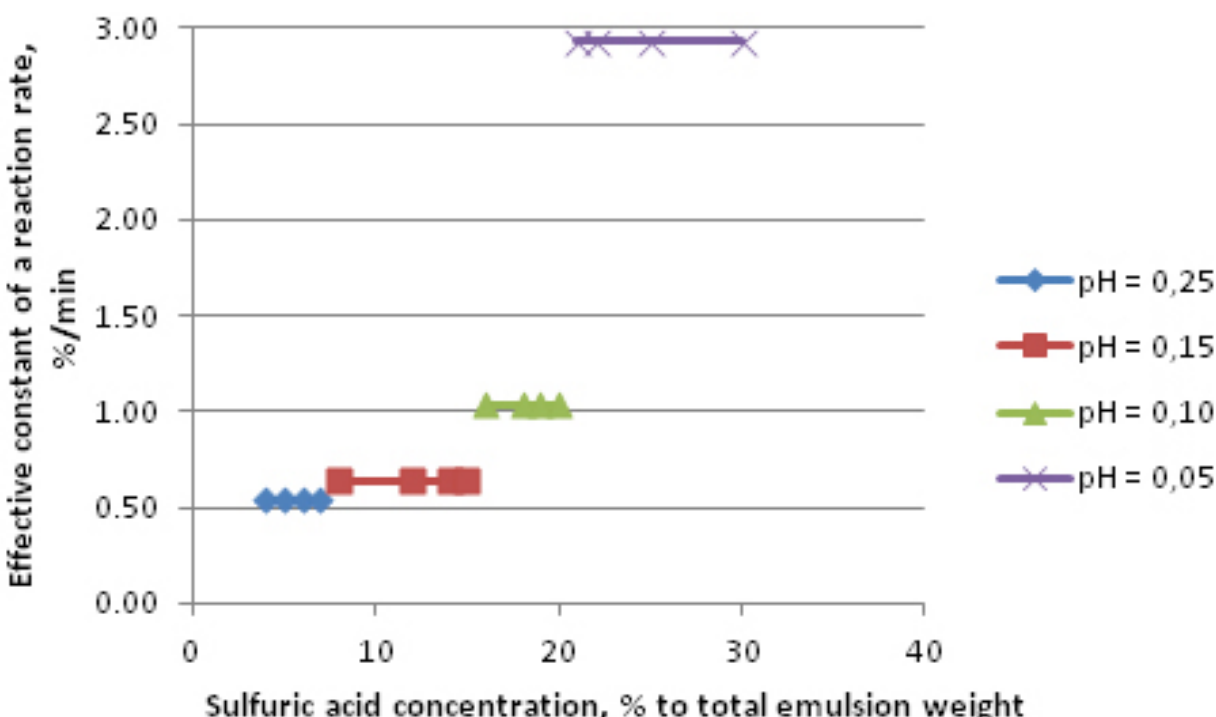

Fig. 3: Dependence of the oxidative decomposition rate effective constant vanadocene dichloride from the concentration of sulfuric acid 
nitric acid may act as electrophilic heterogeneous catalyst and an oxidant. To test this hypothesis was conducted a series of experiments in which acid consumption modified for demetalation process. The results are shown in Table 2.

Thermostating time in all experiments was 3 hours, $\mathrm{pH}$ of obtained productive solutions was 0.25 . The initial concentration of vanadium in oil is $0.35 \%$. Also, an experiment was conducted without the addition of acids using only the electro water. However, in this case no demetallation occurs, vanadium is not found in productive solutions. As a result of experiments it was proved then both acids are used in demetallization process. Formally, this on the one hand indicates that none of the acid is not a catalyst for the process, as catalyst should not be consumed in significant amounts. On the other hand the exhaust gas analysis for the content of sulfur and nitrogen oxides $\left(\mathrm{SO}_{2}, \mathrm{NO}_{2}\right)$ showed the presence of carbon dioxide $\left(\mathrm{CO}_{2}\right)$ obtained in the process of deep oxidation of petroleum hydrocarbons.

Moreover, increasing the concentration of sulfur in the oil under conditions of excess nitric acid proves another side reaction - sulfation of oil hydrocarbons, what also was confirmed by IR spectral analysis of demetallized oil.

The results are given in Figure 2 and 3 in the form of graphs of the extraction degree of vanadium in the solution from a productive time and the reaction rate constant depending on the concentration of the intended catalyst - sulfuric acid on the total weight of the emulsion.

As can be seen from the results of the research of vanadocene dichloride oxidative decomposition kinetics, this process accelerates by specific electrophilic acid catalysis, in which there is a rapid establishment of photolytic equilibriums in solution and subsequent slow conversion by protonated reactant into reaction products.

Taking into account that the sulfuric acid mainly acts as a catalyst and partially as an oxidant, in comparison with nitric acid, $\mathrm{pH}$ variation of final productive solutions made by changing the concentration of sulfuric acid on the weight of the emulsion, and the concentration of nitric acid is taken for constant. The concentration of nitric acid is taken based on the results of preliminary experiments Table $2-4.5 \%$ by weight of the emulsion.

The research of the electrophilic nature of catalysis of viscous oil demetalation process opens up great opportunities to improve this technology. Since the actual process of oil production in the field is conducted through Karajanbas fracturing oil wells out of mining as an emulsion with water to a water content of 30-40\%. Prior demetalation step emulsion must be divided by separation, it is necessary to emulsify it again with water and only then fed the secondary emulsion to the reactor. This technology considerably increases the number of steps, but vanadium concentration reduced in productive solutions, operating costs are rising.

The novelty here is that we offer hydraulic fracturing to produce not by normal water, and warmed electroactivated solution. As a result it is possible to get directly the emulsion, which can be sent to the demetalation reactor after the primary separation.

\section{CONCLUSIONS}

In the result of research it was defined that demetalization of heavy oil of Karazhanbas oilfield proceeds according to electron-seeking mechanism of oxidative reduction of vanadocene dichloride. It is recommended to carry out the process in mixed heterogeneous-heterophase mode with using immobilized catalysts. Very efficiently conduct emulsification with ultrasound at a frequency of $5 \mathrm{MHz}$ at a power of $10 \mathrm{~W} / \mathrm{cm}^{3}$. It will provide the kinetic area medium, and there is no necessity to create turbulized medium for process intensification. It was defined that emulsifying in the system oilelectro activated solution directly in oil-bearing bed is having prospects. It could allow to decrease the number of technological stages and improve the general engineering-and-economical performance of the process. 


\section{REFERENCES}

1. Kopf-Maier P., Kopf H. Vanadocendichlorideinweiteres Antitumor-Agensaus der Metallocen-reihe. 1979, 34-B(6),.805-807.

2. Nadirov N.K., Kotova A.V., Kamyanov V.F., New oil of Kazakhstan and their use: Metals in oil. Alma-Ata, 1984; 448

3. Akhmedzhanov T.K., Nuranbayev G. and others., Patent RK 25065 dated 15.11.2011. Inventor's certificate 2010/1293.1, publ. in 11, dated 15.12.2012, 2012.

4. Batkayev R.I., Nugmanov À.À., Development of complex technologies for recycling of technogenic wastes. Shymkent, 2006; 32

5. Beysenbayev O.K., Isa A.B., Kovaleva A.Y.
Research of Polyacrylonitrile Saponification Heterophase Process Mechanism in Different Conditions// Orient J Chem 2015; 4-9.

6. Vysotskaya N.A., Kabylbekova B. and others. Role of components containing in water on the formation corrosion - scale deposits in pipelines of heating system// Orient $J$ Chem 2015; 261-269.

7. C. Lorber: "Vanadium Organometallics." Chapter 5.01. Comprehensive Organometallic Chemistry III. Elsevier, 2007. 1-60.

8. Christoph Elschenbroich: Organometallchemie. B. G. Teubner Verlag, 2008; 50. 